\title{
Sur les non-maladies et la médicalisation
}

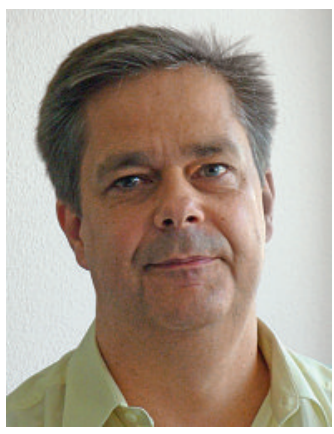

Eberhard Wolff
Pourriez-vous citer spontanément une maladie qui n'en est en fait pas une? En 2002, le British Medical Journal (BMJ) a publié les résultats d'un sondage à ce sujet [1]*. En tête: le vieillissement. La plupart d'entre vous seront d'accord pour dire que le vieillissement n'est pas, à proprement parler, une maladie et pourtant vous ne seriez pas prêts à renoncer totalement à traiter les symptômes liés à l'âge.

Avec ce «hit-parade des non-maladies», le BMJ a voulu d'une part montrer combien le concept même de «maladie» est délicat et d'autre part mettre en lumière la tendance de notre société à vouloir classer toujours plus de troubles comme des maladies. Autre exemple: la dépendance, ancien «péché» devenu «maladie», à l'instar de la «dépendance au sexe» [2] qui fait les choux gras des médias.

Notre société tend en effet à «médicaliser» toujours plus de phénomènes sociaux - en d'autres termes, les interpréter et les aborder sous l'angle médical et les placer dans la sphère d'influence de la médecine. Un thème tout trouvé pour le dernier congrès de l'ASSM/CNE [3], où il a notamment été question du syndrome de carence en fer, devenu un diagnostic à la mode. Car la fatigue est aujourd'hui bien trop rapidement expliquée par une carence en fer, alors qu'elle pourrait avoir beaucoup d'autres origines, médicales ou non. Pire encore: le traitement coûteux utilisé pour pallier cette carence est «en passe de devenir un médicament de confort onéreux» [4].

Cette médicalisation croissante se reflète dans la part des dépenses liées à la santé dans le produit intérieur brut, et plus précisément dans le déplacement de la demande vers les produits de santé, notamment parce que les consommateurs sont rassasiés des autres produits de consommation [5].

L'influence croissante qu'exerce la médecine sur notre quotidien depuis le siècle des Lumières a également été - et est encore - déterminée par des considérations socioculturelles. L'hystérie autour de l'onanie, la peur de la dégénérescence: autant de préoccupations qui traduisent les valeurs de l'époque - tout comme le discours actuel sur le surpoids [6]. Jeunesse, performance et beauté sont aujourd'hui des idéaux pour lesquels la médecine offre toutes sortes d'instruments, et pas seulement les stimulants et le Botox. Parallèlement, la médecine est investie d'une autorité particulière, comme autrefois la religion. Mais c'est précisément là que l'on touche aux limites de la médicalisation: l'autorité des élites médicales (plus vulgairement: les médecins) a passé un pic voilà plusieurs décennies. La dépathologisation de l'homosexualité n'est qu'un exemple de cette démédicalisation active qui va à l'encontre de la tendance générale se dirigeant vers une «société médicalisée» [7].

Mais il serait trop facile de nier leur existence à ces nouvelles maladies en les réduisant à des «maladies à la mode». Au cours des dernières décennies, le «burnout» s'est progressivement établi comme un nouveau concept de maladie, bien qu'il ne puisse être traité au moyen d'un simple médicament, contrairement au TDA/H. L'une des explications possibles est que ce diagnostic permet aux personnes concernées de se sentir comprises et qu'il peut être utilisé comme une métaphore de notre société, comme un moyen de protestation. Mais les gens sont-ils à leur tour médicalisés au moyen du burn-out? La société elle-même se médicalise-t-elle? Ou à l'inverse, est-ce la médecine qui se socialise? Ou alors le phénomène de société que l'on nomme «burn-out» représente-t-il l'occasion de se libérer des schémas de maladies stigmatisants, à l'instar de la dépression [8]?

Une chose est sûre: «la» médecine a gagné en influence. De cette constatation découle tout naturellement la question à l'origine du congrès de l'ASSM/ CNE: où placer les limites de la médicalisation? Les éthiciens et les politiciens aimeraient bien disposer d'outils leur permettant de tracer une frontière claire entre «médicalisation inadéquate» [9] et «médecine réelle», de manière à adapter les catalogues de prestations ou définir des règles en la matière. Il y a quelques décennies, la recherche aurait encore été en mesure de fournir des réponses plus claires. La médicalisation était alors mal perçue car ressentie comme une prise de pouvoir des médecins, au détriment de la société. Le bien et le mal étaient mieux séparés. A présent, la médicalisation est plutôt considérée comme une conséquence de notre mode de vie, comme quelque chose de normal, de désiré. Les exemples présentés lors du congrès, dont le burn-out (ou encore l'augmentation des performances), montrent plutôt qu'il ne peut y avoir de «réelle» médecine sans influence de la société et que les frontières sont bien trop floues et les domaines bien trop imbriqués pour fixer des limites claires. Tout comme le vieillissement n'est pas une maladie - mais, dans un certain sens, si: un concept délicat et difficile à appliquer.

Eberhard Wolff** 


\section{Références}

1 Smith R. In search of «non-disease». BMJ 2002;324:883-5.

2 http://blog.tagesanzeiger.ch/mamablog/index. php/17761/mythos-sexsucht/

3 «La médecine tous azimuts - Problèmes éthiques de la médicalisation du quotidien», symposium organisé par l'Académie Suisse des sciences médicales (www.samw.ch) et la Commission nationale d'éthique (www.nek-cne.ch). Programme et présentations disponibles à l'adresse www.samw.ch/fr/Projets/ Economicisation-medecine.html. Le présent article a été en grande partie inspiré par les conférences du symposium. La radio DRS a également réalisé un reportage sur ce dernier, disponible à l'adresse www. drs.ch/www/de/drs/sendungen/echo-der-zeit/2646. sh10185046.html
4 Cf. exposé de Franziska Demarmels Biasiutti, Berne, à l'occasion du symposium. Roch Ogier, Genève, en revanche, a notamment souligné que la carence en fer n'est pas une nouveauté médicale, mais que les moyens diagnostiques et thérapeutiques existent.

5 Cf. exposé de Stefan Felder, Bâle, à l'occasion du symposium.

6 Cf. exposé d'Iris Ritzmann, Zurich, à l'occasion du symposium.

7 Cf. exposé de Peter C. Meyer, Winterthour, à l'occasion du symposium.

8 Cf. exposé de Peter Schneider, Zurich, à l'occasion du symposium et la discussion qui a suivi.

9 Cf. exposé de Christian Kind à l'occasion du symposium. 